\title{
De Lamarck a Darwin: ¿Continuidad o Ruptura? ¿LINEALIDAD O BIFURCACIÓN?
}

\author{
FROM LAMARCK TO DARWIN: CONTINUITY OR RUPTURE? \\ LINEARITY OR BIFURCATION? \\ Julio Alejandro Castro Moreno*
}

\begin{abstract}
Resumen
En este escrito se hace una comparación entre las teorías de Lamarck y Darwin, para mirar hasta qué punto hay una continuidad entre ellas o, por el contrario, si existe una marcada discontinuidad. Dicha comparación se hace teniendo en cuenta algunos aspectos clave de las dos teorías, tales como las concepciones de especie, extinción, origen de la vida y naturaleza. Así mismo se analizan las formas de proceder de estos dos grandes naturalistas.

Palabras clave: Historia de la ciencia, construcción de analogías y metáforas, evolución y transformación, ruptura y continuidad, azar y necesidad.
\end{abstract}

\section{Abstract}

In this paper we make a comparison between the theories of Lamarck and Darwin, to look if there is continuity among them or, on the contrary, if there is a marked discontinuity. This comparison is made keeping in mind some key aspects of the two theories, such as the conceptions of species, extinction, origin of the life and nature. Likewise we analyze the procedure of these two big naturalists.

Key words: History of the science, construction of analogies and metaphors, evolution and transformation, rupture and continuity, chance and necessity.

\footnotetext{
*Universidad Pedagógica Nacional. bioaleko@latinmail.com
} 
Todo hombre instruido y observador advierte que nada resulta constante en la superficie del globo terrestre. Con el tiempo todo cambia en él y sufre mutaciones diversas más o menos rápidas, según la naturaleza de los objetos y de las circunstancias. Los lugares elevados se degradan perpetuamente por las acciones alternativas del sol, de las aguas pluviales y otras causas todavía. Todo lo que se desprende del suelo es arrastrado hacia los lugares bajos. Los lechos (...) de los ríos, de los mismos mares varían en su forma, su profundidad e insensiblemente se desplazan. En una palabra todo en la superficie de la tierra cambia de situación, de forma, de naturaleza y de aspecto. Hasta los mismos climas de sus distintas comarcas no resultan estables.

Filosofia zoológica Jean Baptiste Lamarck

\section{INTRODUCCIÓN}

En general, los autores que escriben sobre temas relacionados con la biología y que hacen énfasis en la teoría de evolución dedican a lo sumo un capítulo para tratar este importante concepto, pero casi sin excepción, como afirma Gould (1999: 272), empiezan a exponer las ideas lamarckianas para dar paso luego a la postura darwinista. A esto se le suma la utilización del famoso ejemplo del alargamiento del cuello de la jirafa (Gould, 1993b: 153), lo cual pedagógicamente no es oportuno, ya que los estudiantes asumen que fue necesaria la teoría de Lamarck para que, a partir de allí, Darwin construyera la suya. Es decir, que los maestros y los autores de los libros conciben que para conocer la teoría de la selección natural es primordial comprender con antelación la obra de Lamarck.

El propósito de este escrito es hacer una comparación de las teorías de Lamarck y Darwin, para luego dictaminar hasta dónde hay una continuidad entre ellas 0 , por el contrario, si existe una verdadera ruptura entre estas dos formas de ver la transformación de los organismos a través del tiempo. Para hacer esto podría haber utilizado nuevamente el ejemplo del cuello de la jirafa, pero no lo voy a hacer así. Más bien, voy a utilizar una analogía que tiene que ver con dos formas opuestas de hacer la historia de las ciencias, en comparación con las teorías de estos dos grandes naturalistas. Pero dicha analogía la explicaré más adelante; ahora quisiera argumentar por qué no utilizar el cuello de la jirafa como punto de comparación.

\section{El CUELLO DE LA JIRAFA: UNA PUGNA INEXISTENTE}

Quien no haya leído la obra de Lamarck, específicamente su Filosofía zoológica, podrá creer que este naturalista basaba sus explicaciones en el alargamiento del cuello de la jirafa, cuando en realidad es un ejemplo más, al que le dedica unas cuantas líneas, ni siquiera una página de su obra. Podría pensarse también que Darwin usó el ejemplo de este mamífero para argumentar su teoría; a decir verdad sí lo hizo pero no de la manera en que nos lo imaginamos. 
Leamos ahora al propio Lamarck acerca de su tan citado ejemplo:

Relativamente a los hábitos, es curioso observar el producto en la forma particular y talla de la jirafa. Se sabe que este animal, el más alto de los mamíferos, vive en el interior de África, donde la región árida y sin praderas le obliga a ramonear los árboles. De este hábito, sostenido después de mucho tiempo, en todos los individuos de su raza, resultó que sus patas delanteras se han vuelto más largas que las de atrás y que su cuello se ha alargado de tal manera que el animal, sin alzarse sobre las patas traseras levanta su cabeza y alcanza con ella a seis metros de altura (Lamarck, 1986 [1809]: 187-188).

El anterior párrafo es todo lo que Lamarck escribió acerca de las jirafas. Por tanto, es posible inferir que no es una parte central de su teoría. Además de esto, es posible percibir un error de observación en su ejemplo: las patas delanteras de este animal tienen la misma longitud que las traseras. Si Lamarck se consideraba tan buen observador, ¿por qué cometió este error tan garrafal? $\mathrm{Si}$ se quisieran citar ejemplos más elaborados yo escogería el de las serpientes. Lamarck afirma que estos animales debieron tener patas en la antigüedad, pero las perdieron por el hábito de arrastrarse: "su cuerpo, por efecto de los esfuerzos repetidos para alargarse, con el fin de pasar por espacios angostos, adquirió una longitud considerable y no proporcionada a su anchura" (Lamarck, 1986 [1809]:
181). Como las patas no cumplían una función en un cuerpo tan largo, entonces desaparecieron.

De otro lado, Darwin citó, en la primera edición de El origen de las especies, un ejemplo relacionado con la jirafa, pero no concerniente al cuello, sino a la cola (Gould, 1999: 276), la cual había sido seleccionada por ser un buen espantamoscas, evitando así que estos insectos mitigaran la fuerza del mamífero, impidiéndole que colonizara otros territorios o haciéndolo más vulnerable para los depredadores. En su libro La variación de los animales y las plantas en estado doméstico, Darwin argumentó que el cuello de la jirafa no pudo evolucionar independientemente de las otras partes del cuerpo, pero que este proceso no puede ser al unísono, sino que una parte evoluciona primero y luego lo empiezan a hacer las otras, gradualmente. En la última edición de su obra cumbre, Darwin retomó el ejemplo del cuello de la jirafa para responderle a alguno de sus críticos, y puede que allí, según Gould (1999: 280), esté "una fuente primaria para la leyenda [Darwin contra Lamarck con respecto a la jirafa] tal como se desarrolló posteriormente".

Dicha leyenda se hace todavía más insostenible cuando nos percatamos de que Darwin defendía ciertos conceptos lamarckianos para sostener la evolución de algunos seres, ya que "el propio Darwin (...) no negó el principio lamarckiano de herencia de los caracteres adquiridos por el uso o perdidos por desuso. Consideró que el mecanismo lamarckiano era débil, infrecuente y completamente 
subsidiario a la selección natural, pero aceptó la evolución por el uso y el desuso" (Gould, 1999: 280-281).

Como podemos ver, de un lado, Lamarck no utilizó el ejemplo del alargamiento del cuello de la jirafa como una parte central de su argumentación y, de otro lado, Darwin no explicó el alargamiento del cuello de la jirafa (en las pocas veces que intentó hacerlo) en términos que hoy podamos llamar darwinistas, es más, utilizó y defendió conceptos de la teoría de Lamarck para explicarlo. Es muy probable que los evolucionistas de la actualidad no puedan explicar este famoso ejemplo de una manera totalmente satisfactoria, ya que el cuello de la jirafa, tal vez, evolucionó debido a otras causas no relacionadas con su uso actual (Gould, 1999: 281): por ejemplo, se sabe que los machos luchan usando sus cuellos y sus cabezas, y que sus combates en ocasiones pueden ser mortales. ¿Hasta qué punto habrá evolucionado esta parte del cuerpo de dicho animal por medio de la selección sexual? Puede que sea tan improbable como por medio de la herencia de los caracteres adquiridos 0 por el uso.

Podríamos concluir de todo lo que se ha dicho hasta ahora que no hubo un verdadero conflicto entre Lamarck y Darwin en cuanto al ejemplo de la jirafa. Parece ser que éste se ha convertido en una leyenda y en una herramienta pedagógica supuestamente efectiva, lo cual no es así. Es muy factible que este ejemplo haya sido heredado generación tras generación (de libros) y que se haya forta- lecido gracias al uso excesivo que se la ha dado. Pero dejemos en paz de una vez por todas a la jirafa, ya que, como veremos, el conflicto Darwin-Lamarck es mucho más complejo de lo que parece, $y$ para poder hacer una explicación de dicho conflicto es necesario que nos detengamos a exponer la teoría de Lamarck, para luego compararla con la de Darwin en algunos puntos fundamentales. Es de anotar que, en otro trabajo, hemos hecho un análisis de la teoría darwinista (Castro, 2004); por tanto, en el siguiente aparte nos detendremos a explicitar la teoría lamackiana.

\section{LA TEORÍA DE LAMARCK}

Para nadie que haya estudiado acerca del tema de la evolución es un misterio que Lamarck pasó a la posteridad con más pena que gloria, con respecto a su teoría publicada en 1809. En muchos círculos científicos y académicos se toman sus ideas como un error del pasado o a él mismo como el que abrió las puertas hacia la teoría de la evolución para que el glorioso Darwin entrara a través de ellas. Si bien es cierto que prácticamente toda la teoría lamarckiana ha sido echada al piso, también es cierto que este personaje fue el primero en proponer un mecanismo que explicase la transformación de los seres a través de largos periodos de tiempo: "Lamarck ofreció (...) la mejor teoría sobre el mecanismo de la evolución a la que alguien pudiera haber llegado en aquél tiempo (...) ha sido una desgracia (...) el que, por lo menos en el mundo de habla inglesa, su nombre se 
haya convertido en sinónimo de un error -su teoría sobre el mecanismo de la evolución- más que de su creencia correcta en el hecho de que la evolución tuvo lugar" (Dawkins, 1988: 220). Y este mecanismo, creámoslo o no, estaba basado en leyes naturales, no en milagros.

Aunque sus ideas sobre la transformación han pasado a la historia, es pertinente recordar que Lamarck fue uno de los naturalistas que acuñaron el término Biología Biología a esta ciencia naciente, pero no solamente ideó el término, sino que descubrió en lo vivo un problema de conocimiento, dándole gran importancia a la organización de los seres. De otro lado, aunque estudió botánica, fue quien ideó el término invertebrados para los animales sin vértebras e hizo importantes estudios sobre ellos. Según Smith, es muy probable que las ideas de la transformación en Lamarck hubiesen nacido "durante los años que pasó clasificando y anatomizando las inmensas colecciones de invertebrados que poseía en el museo (Musée d' Histoire Naturelle)" (Smith, 1997: 334). Estas ideas son las que pretendo comentar a continuación.

Ernst Haeckel, en el prólogo de una edición de la Filosofía Zoológica (Lamarck, 1986 [1809]: vi-vii) enumera algunas de las proposiciones de la teoría lamarckiana:

- Las divisiones sistemáticas son un producto artificial de la humanidad.

- Las especies (razas) no son contemporáneas, pues descienden unas de otras y sólo poseen una fijeza relativa y temporal.
- Las variedades engendran las especies.

- Las modificaciones en el medio influyen en los organismos: sobre la organización, la forma general y los órganos. Lo mismo puede decirse del uso y del desuso.

- Se han producido en primer lugar los animales y plantas más sencillos (menos complejos), después nacieron los seres de organización más compleja.

- La evolución geológica y la repoblación del planeta ha ocurrido de una manera no catastrófica. Han ocurrido continuamente y sin interrupciones violentas.

- La vida no es más que un fenómeno físico.

- Todos los fenómenos vitales son manifestaciones mecánicas (físicas o químicas) que tienen razón de ser en la constitución de la materia orgánica.

- Los seres más primitivos han nacido y nacen por generación espontánea.

- Todos los cuerpos vivientes están sometidos a las mismas leyes que los seres privados de vida.

- Las ideas y demás manifestaciones del espíritu son debidas a movimientos que se producen en el sistema nervioso.

- Las características adquiridas por un individuo son heredadas a su descendencia.

Aunque son muchas las ideas en la obra de Lamarck, en general han sido reducidas a dos principios fundamentales: el primero de ellos es el del uso y el des- 
uso, lo cual quiere decir que si un órgano es usado con frecuencia, éste se volverá más fuerte, se desarrollará en proporciones mayores que los demás, pero si, por el contrario, no se usa con regularidad, éste se atrofiará e incluso puede llegar a desaparecer. El otro principio de la teoría lamarckiana es el de la herencia de los caracteres adquiridos, el cual consiste en que si un individuo adquiere una característica, ésta pasará a la siguiente generación: por ejemplo, si un hombre es herrero y tiene un hijo, éste heredará no sólo la profesión de su padre, sino también los brazos fuertes.

Es curioso ver cómo estos dos conceptos, que se cree fueron una invención de Lamarck, eran en realidad dos hechos que hacían parte de la cotidianidad de las personas en su época. En el caso del uso y el desuso, esto se puede corroborar mediante la siguiente cita, escrita en 1802 (antes de que Lamarck publicara su teoría) por el teólogo William Paley, en la que quiere desvirtuar el efecto del uso y el desuso:

Las mamas del macho no han desaparecido por falta de uso; nec curtorum, per multa saecula, Judaeorum propagini deest praeputium [ni el prepucio de los judíos se ha acortado en su descendencia después de tantos siglos de circuncisión] (Gould, 1994: 136).

De lo anterior podemos concluir que el principio del uso y del desuso no es una invención de Lamarck, sino una idea de sentido común en su tiempo. Igualmente, según Gould (1986: 80-82), Lamarck creía en la herencia de los caracteres adquiridos, pero no era una idea original suya, ni constituía el eje central de su teoría. Muchos científicos releyeron a Lamarck y sobredimensionaron el papel de este principio para hacer contrapeso a la teoría de Darwin. Desde este punto de vista, la herencia de los caracteres adquiridos sí cumple un papel importante en la teoría lamarckiana, pero no es el papel protagónico, es el mecanismo que garantiza que los hijos se beneficien de los logros biológicos de los padres, pero no es el motor que impulsa a los seres a ascender por la escalera. Es paradójico ver cómo la herencia de los caracteres adquiridos no explica la evolución biológica, pero se aplica perfectamente a la evolución cultural. Es igualmente irónico que el mismo Darwin creyera en estos dos postulados y en cierto modo los defendiera, aunque ellos no hayan conformado una parte importante en su teoría: no hacían parte de su mecanismo.

Como ya vimos, la teoría de Lamarck no se puede reducir a estos dos conceptos. Trataré ahora de resumirla intentando abarcar otras de sus ideas fundamentales:

En primer lugar, Lamarck afirma que los animales (y las plantas) son producciones de la naturaleza, que no han sido creados todos los seres al mismo tiempo, sino que primero surgieron los más sencillos (por generación espontánea) y que fueron ascendiendo por la gran cadena del ser hacia el hombre. Para que esto ocurra, la naturaleza dispone de las circunstancias y del tiempo: : Todo cambio en las circunstancias en que se encuentra un animal (clima, geografía, modos 
de vida, etc.) y el mantenimiento de este cambio por un espacio considerable de tiempo hacen que se cree una modificación en las necesidades de dicho animal, $y$ "que todo cambio en las necesidades de los animales produce en ellos nuevas acciones para satisfacerlas, y por consecuencia otros hábitos" (Lamarck, 1986 [1809]: 174). Además, la satisfacción de una nueva necesidad, implica que el animal lleve a cabo nuevas acciones, por lo cual debe hacer un uso más frecuente de algunos de sus órganos, lo que lleva a que se desarrollen y se fortifiquen.

Aquí es evidente que para que un animal se transforme es necesario, de un lado, un cambio en las circunstancias y, de otro, un esfuerzo del animal por realizar acciones que le permitan adquirir nuevos hábitos $\mathrm{y}$, por ende, desarrollar algunas partes de su cuerpo, para así hacer frente a las nuevas condiciones de vida.

La expresión: las circunstancias influyen sobre la forma y la organización de los animales no se debe tomar al pie de la letra porque, según Lamarck, se le atribuiría un error, ya que las circunstancias no operan directamente sobre la forma y la organización; no producen sobre ellas ninguna modificación. Pero si las circunstancias cambian considerablemente afectan las necesidades de los animales y de esta manera sus acciones, sus hábitos $\mathrm{y}$, por último, la forma y organización de estos seres.

La teoría de Lamarck se puede esquematizar de manera muy sencilla con la siguiente figura.

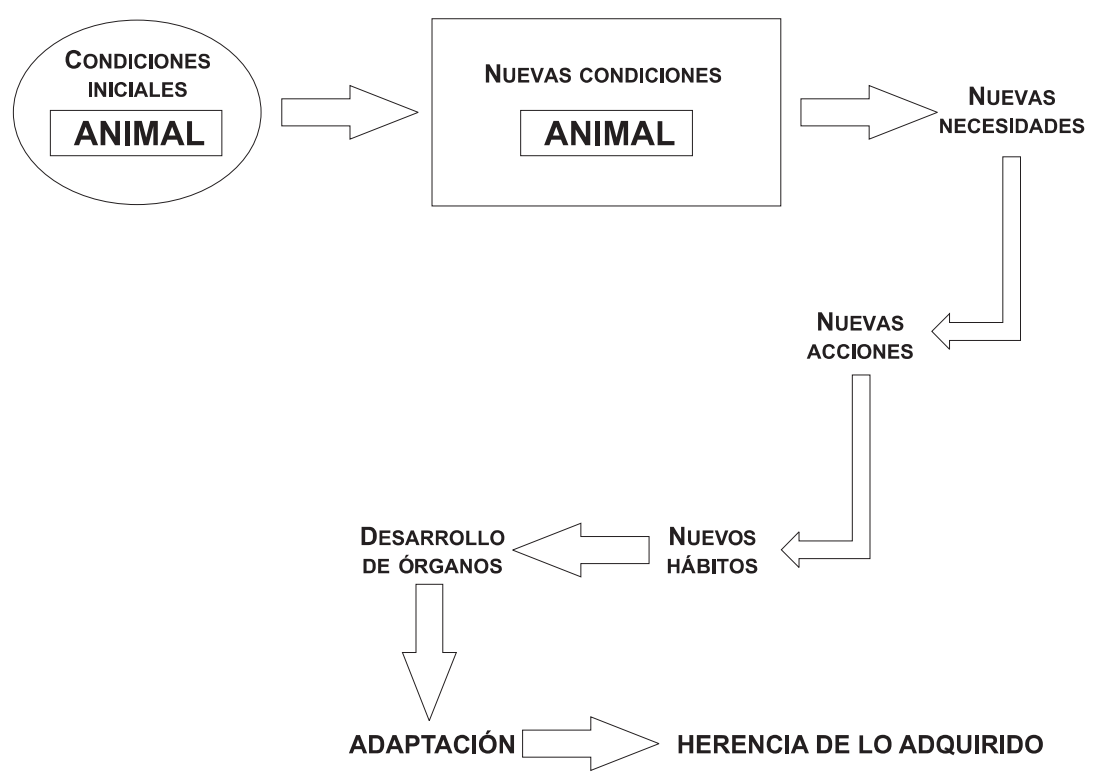


Una vez que Lamarck explica el mecanismo de su teoría propone lo que él llama las dos leyes de la naturaleza, que se enuncian a continuación (Lamarck, 1986 [1809]: 175):

\section{Primera Ley:}

En todo animal que no ha traspasado el término de sus desarrollos, el uso frecuente y sostenido de un órgano cualquiera lo fortifica poco a poco, dándole una potencia proporcionada a la duración de su uso, mientras que el desuso constante de tal órgano le debilita y hasta lo hace desaparecer.

\section{Segunda Ley:}

Todo lo que la Naturaleza hizo adquirir o perder a los individuos por la influencia de las circunstancias en que su raza se ha encontrado puesta durante largo tiempo, y consecuentemente por la influencia del empleo predominante de tal órgano, o por la de su desuso, la Naturaleza lo conserva por la generación en los nuevos individuos, con tal de que los cambios adquiridos sean comunes a los dos sexos, o a los que han producido estos nuevos individuos.

Vemos en estas dos leyes, los dos principios por los que se ha identificado la obra de Lamarck, pero como he argumentado líneas atrás no podemos reducir el lamarckismo a estos dos principios, ya que su teoría es más amplia de lo que parece. Lo que he intentado exponer en las últimas líneas es la importancia que tienen para Lamarck el tiempo, las circunstancias y los esfuerzos de los organismos por adaptarse. Desde su punto de vista, "las fuerzas transformadoras esenciales eran las circunstancias favorables y el tiempo, actuando sobre el organismo maleable; no había límites para el tiempo, y las circunstancias eran siempre cambiantes" (Coleman, 1985: 118-119).

Como síntesis de este aparte, es preciso mencionar que el estilo utilizado por Lamarck para escribir su teoría (especialmente su noción de "marcha de la naturaleza") está notablemente influenciado por el clima intelectual de su época: el siglo de las luces. De otro lado, es interesante ver que Lamarck, en su teoría, concibe la transformación espaciotemporal de los organismos mediante la intervención de dos tipos de fuerza: una interna o "energía vital" inherente a la vida, que se puede evidenciar en lo que él denomina como esfuerzos de los individuos $\mathrm{y}$, una externa, de las circunstancias, que se traduce en la respuesta de los individuos a los cambios en dichas condiciones. Como consecuencia de estas fuerzas, los organismos, después de muchas generaciones, se transforman radicalmente, diferenciándose así de sus progenitores.

Sin embargo, hay otros aspectos de dicha teoría que es necesario resaltar, pero lo haré más adelante, cuando argumente con mayor claridad cuáles son las diferencias de fondo entre las ideas de Darwin y Lamarck. 


\section{DARWIN FRENTE A LAMARCK: UNA ANALOGÍA DE LA HISTORIA DE LAS CIENCIAS}

Desde el punto de vista de Jacob (1986) hay dos formas de hacer la historia de las ciencias:

Una de ellas es ver la historia como algo lineal. Una teoría da origen a otra, se transforma: una idea poco a poco se adapta a su contexto y se transmuta en una idea más elaborada. En suma, esta forma de hacer la historia de las ciencias es concibiéndola como una "sucesión de ideas y como la genealogía de éstas" (Jacob, 1986: 10). Las ideas de este tipo de historia se comportan como los seres vivos: nacen, producen hijos y mueren; pero sus hijos dan cierto tipo de continuidad. De acuerdo a este tipo de historia, "se puede ver en Lamarck al precursor de Darwin; en Bufón, al de Lamarck (...) y así sucesivamente" (Jacob, 1986: 11). A este tipo de historia la llamaré Historia lamarckista, porque concibe el progreso o evolución de las teorías científicas del mismo modo que Lamarck concibe la transformación de los seres vivos. La forma de representar este tipo de historia es la línea o la columna.

La otra forma de hacer la historia de la biología, y de la ciencia en general, es "investigar cómo los objetos han llegado a hacerse accesibles al análisis" (Jacob, 1986: 11), generando así las condiciones para que las disciplinas se transformen en nuevas ciencias. Lo que hay que hacer es estudiar la naturaleza de dichos objetos de investigación, "la actitud de quienes los estudian, su manera de o bservar, los obstáculos que levanta frente a ellos su cultura" (Jacob, 1986: 11). Ya las ideas no se unen como los hijos a los padres, ya no hay una linealidad en el tiempo. Desde este punto de vista, "Darwin ya no es simplemente el hijo de Lamarck y el nieto de Buffon" (Jacob, 1986: 12). Denominaré a este tipo de historia como Historia darwinista, y su forma de representación es el árbol. Pero no es un árbol cualquiera: cada rama puede sufrir muchas bifurcaciones; en algunas de ellas se verán zarcillos, que se enredan de una manera muy difícil de descifrar; en otras ramas pueden aparecer flores o inflorescencias; en otro tipo de ramas se podrán observar diversos tipos de frutos; y otras desdichadas se verán marchitas con sus hojas secas. Así mismo, las ramas se podrán entretejer de diversas maneras, algunas producirán más bifurcaciones que sus vecinas y otras se romperán, quedando en el olvido.

Este árbol de la historia estará creciendo constantemente y en algunos casos no sabremos de qué rama proceden otras. Aunque hay una continuidad en el tronco principal (el tiempo es irreversible) en las diferentes ramificaciones hay una ruptura evidente. Escogí el árbol como modelo porque se ajusta a la explicación darwinista de la evolución: recuérdense los árboles filogenéticos. Así como hay discontinuidades o rupturas en el registro geológico, pueden hallarse rupturas en la historia de las ciencias. Así como hay especies extintas y hay "eslabones perdidos" entre los organismos, de igual modo 
puede haber en la historia de las ciencias ideas extintas. Es necesario aclarar que, si bien se asume la existencia de un tronco común para todas las ramas, desde nuestra analogía es imposible concebir que todas las ideas científicas procedan de un único tronco y que, además, ellas se ven notablemente influenciadas por diferentes formas de ver el mundo, es decir, por el contexto sociocultural.

He utilizado esta analogía con el fin de explicar que entre Lamarck y Darwin no hay un gradualismo (principio que los dos defendían), sino una ruptura. Podría decirse que no era necesario que existiera la teoría de Lamarck para que pudiera surgir la obra de Darwin... jesto es un anacronismo!, ya que la historia estudia lo que ha ocurrido, cómo y por qué ha acontecido, no lo que pudo ocurrir.

Además de la discontinuidad que se argumentará más adelante, hay una nueva situación a tener en cuenta. Desde el punto de vista de Dawkins (1988), el lamarckismo es una teoría rival del darwinismo. Evidentemente, la teoría del francés apareció medio siglo antes que la del inglés (podría verse aquí otro anacronismo): lo que se quiere decir es que algunos científicos contemporáneos de y posteriores a Darwin, releyeron y defendieron la obra de Lamarck para oponerse férreamente a la teoría de la selección natural.

Aunque hay evidencias de que Darwin conoció el trabajo de Mendel (Vorzimmer, 1968), es de resaltar que no los tuvo en cuenta (como hizo la mayoría de sus colegas). De otro lado, es interesan- te notar que, aunque Darwin creía en el uso y el desuso, así como en la herencia de los caracteres adquiridos, en su autobiografía calificaba de disparates las ideas de Lamarck, como la "tendencia al progreso" y "las adaptaciones debidas a las paulatinas inclinaciones de los animales". A pesar de ello, Darwin reconocía que las conclusiones de ambos eran muy similares, pero que llegaron a ellas por diferentes vías (postularon mecanismos diferentes). En una nota al pie, en su bosquejo histórico de El origen de las especies escribió: "Es curioso observar cuánto anticipó mi abuelo, el Dr. Erasmo Darwin, las opiniones y los erróneos fundamentos de Lamarck en su Zoonomía (...) publicada en 1794" (Darwin, 1986 [1859]). Pero no sólo hubo críticas negativas: en un aparte de El Origen, Darwin concibe a Lamarck como el primer naturalista en concluir que todo cambio en el mundo orgánico como en el inorgánico está sujeto a leyes naturales y no a causas sobrenaturales o milagros.

Para poder ver los aspectos en que hay una ruptura, compararé algunos conceptos y procederes en estos dos naturalistas. En cuanto a los conceptos están los siguientes: La naturaleza, el tiempo, las especies, las extinciones y el origen de la vida. En cuanto a los procederes tendré en cuenta el método que siguieron para llegar a sus conclusiones.

\section{LA NATURALEZA}

Un avance fundamental en la teoría lamarckiana, es la importancia que le da a la naturaleza en cuanto a la producción 
de los seres vivos. Sin embargo, es muy difícil comprender el significado que le da a ella, por tanto, lo que expondré a continuación podrá caer en una especulación profunda. De un lado, hace referencias a leyes de la naturaleza, que son rígidas, susceptibles de ser observadas y comprendidas (en esto no difiere mucho de Darwin). Pero asume que la naturaleza tiene un plan definido y que la culminación del progreso de los organismos es el ser humano. En esta intencionalidad es en donde realmente hay una ruptura con Darwin, ya que él no ve ninguna intención, ninguna inteligencia que guíe el curso de la naturaleza. Aunque Lamarck intente demostrar que la naturaleza actúa de acuerdo a sus propias facultades, en algunos apartes de su obra menciona que la naturaleza fue dotada de estas facultades por medio de su Autor (¿Dios?).

Esto queda demostrado en el siguiente párrafo: se cree que las especies son tan antiguas como la naturaleza y habían sido creadas por el "Supremo Autor de Todo lo que existe. Nada existe, en efecto, sino por su voluntad; pero, ¿podemos asignarle reglas en la ejecución de ella *la naturaleza* y fijar el modo que ha seguido a este respecto? ¿Su poder infinito no pudo crear un orden de cosas, que diese sucesivamente la existencia a todo lo que vemos como a todo lo que existe y no conocemos?" (Lamarck, 1986 [1809]: 52). Posteriormente afirma que él respeta la forma de proceder del Autor y que no se inmiscuye en ello, sólo se limita a observar la naturaleza. Si de esto se vislumbra la marcha de ella para producir cosas, asegura poder entender la voluntad de su Autor.

Giordan (1988: 171), respecto a las ideas de Lamarck, caracteriza la intervención de Dios en la naturaleza de la siguiente manera:

En realidad, la naturaleza debe seguir leyes rígidas, leyes que no puede cambiar. Éstas han sido creadas por Dios, pero Dios (concepto que conserva por diversas razones) no ha creado ni los animales ni las plantas. Lamarck ha hecho que Dios retrocediera un paso: de creador directo de las plantas y los animales, pasa a ser únicamente el creador de la naturaleza, y es esta la que, a su vez, produce los seres vivos.

De lo anterior podemos inferir que, si bien la naturaleza actúa bajo leyes, éstas han sido ideadas por un ser supremo, mientras que Darwin destrona cualquier intervención sobrenatural en el devenir de la naturaleza. Además de ello, Lamarck sitúa las condiciones y el tiempo por fuera de la naturaleza; son sus ayudantes, sus herramientas, de igual modo los seres vivos son producciones de la naturaleza no son naturaleza. Darwin concibe el tiempo, las circunstancias y los seres vivos como parte de la naturaleza; esto queda demostrado cuando explica la selección natural. Como es la naturaleza la que selecciona, los seleccionadores, por ejemplo, pueden ser seres vivos como los depredadores o los parásitos, o un agente no vivo como un cambio climático.

Lamarck ve la muerte de algunos individuos como parte del equilibrio de la 
naturaleza, para mantener constante el número de seres que habitan el planeta:

Por consecuencia de la extrema multiplicación de las pequeñas especies, y especialmente de los animales más imperfectos, la multitud de los individuos podría perjudicar a la conservación de las razas, a la de los progresos adquiridos en el perfeccionamiento de la organización, en una palabra, al orden general, si la Naturaleza no hubiera tomado precauciones para restringir esta multiplicación en límites que no puede franquear nunca (Lamarck, 1986 [1809]: 83).

Para Lamarck era evidente que las especies fuertes devoran a las débiles, que rara vez se devoran entre sí los seres de una misma raza, puesto que están empeñados en "hacer la guerra a los de otras razas". La excesiva multiplicación de algunas especies (sobre todo las más sencillas) haría inhabitable el planeta, si la naturaleza no se hubiera ideado la forma de mantener a raya esta multiplicación (haciendo que se devoren unos a otros). De ahí que el número de individuos se mantenga constante. Según Lamarck, solamente el hombre se escapa a esta doctrina (no es devorado por otros) y se multiplica de una manera muy efectiva. Sin embargo, la naturaleza lo dotó de pasiones negativas que lo empujan a atacar y destruir a sus semejantes (¿guerra?). De este modo la tierra nunca estará sobrepoblada y todo lo que parece desorden o anomalía no es más que orden: el equilibrio de la naturaleza.

Pero allí donde Lamarck ve un equi- librio constante, como expresión de la voluntad de la naturaleza (o su Autor), Darwin no ve otra cosa que competencia, lucha por la existencia.

\section{LAS ESPECIES}

Según Lamarck, las especies no existen en la naturaleza, es más, cualquier categoría taxonómica no existe en ella, lo único que existe son los individuos; todo lo demás es artificial, y aunque nos ayudan a organizar el conocimiento no son entidades reales, no son producciones de la naturaleza. Una de las formas de argumentar ello es diciendo que las especies no son constantes sino en un espacio delimitado de tiempo, las especies son mutables y no hay una demarcación fija entre una especie y otra: prueba de ello es que se pueden producir híbridos; las especies no están totalmente demarcadas. Desde este punto de vista, los géneros, familias, clases, etc., no existen de ningún modo. Lamarck ataca la doctrina de las especies de la siguiente manera:

La suposición casi generalmente admitida de que los cuerpos vivientes constituyen especies constantemente distintas por caracteres invariables, $y$ que la existencia de ellas es tan antigua como la de la propia Naturaleza, fue establecida en un tiempo en que faltaban los medios de observación y en el que las ciencias naturales resultaban casi nulas (Lamarck, 1986 [1809]: 51).

Lo que puede ser similar a la postura darwinista es el hecho de que las especies no son tan antiguas como la naturaleza. 
El concepto de especie en Darwin es mucho más complejo de definir. Lo que se puede suponer es que Darwin asumió la existencia de las especies, ya sea como entidades reales (naturales) o como entidades abstractas, pero mucho más constantes que en Lamarck. Si no fuera así, le hubiera puesto otro título a su obra más reconocida, o ni siquiera se hubiera planteado el problema de cómo se originan las especies.

Lo que sí es cierto es que Darwin tenía muchos problemas para diferenciar las variedades de las especies, entre otras cosas porque variedad tenía una connotación muy diferente en botánica, a la que él usaba con las razas de animales. Prueba de esta confusión es el siguiente pasaje:

Hace muchos años, cuando yo comparaba y veía comparar a otros las aves de las islas vecinas del archipiélago de las Galápagos, unas con otras, y las mismas con las de tierra firme americana, me sorprendió mucho ver cuán vaga y arbitraria era la distinción entre especies y variedades (Darwin, 1986 [1859]: 61-62).

Pero la confusión de Darwin no ocurrió solamente durante su viaje. Una vez en tierra firme, el ornitólogo John Gould le comunicó que los ejemplares de sinsontes de las Galápagos no eran pertenecientes a variedades similares, sino a especies deferentes, pero muy parecidas. De lo anterior, Darwin se aferró aún más a la inestabilidad de las especies (Gould, 1993a: 303).
De acuerdo a las argumentaciones de Mayr (1992: 40-44), en la época de Darwin existían 4 conceptos de especie:

- Tipológico, es una agrupación constante, inmutable y fija.

- Nominalista, sólo existen individuos que por similitud se agrupan bajo un nombre.

- Evolutivo, es un concepto paleontológico, conjunto de poblaciones ancestrales-descendientes.

- Biológico; las especies son grupos de poblaciones naturales con cruzamiento entre sí, que están aislados reproductivamente de otros grupos.

Según Mayr (1992), en los primeros escritos de Darwin (notas) se observa que él asumía un concepto tipológico de especie, para luego ir asumiendo uno biológico, que mantuvo por cerca de 15 años, pero debido a la dificultad de diferenciar especies de variedades, al escribir su teoría se identificó con un concepto de especie nominalista-tipologista, que es evidente en la primera edición de El origen de las especies:

Los sistemáticos... no serán incesantemente perseguidos por la duda sombría de si esta o aquella forma son especies en esencia. Estoy seguro de que esto ... no supondría ningún alivio ... Los sistemáticos sólo tendrán que decidir ... si una forma es lo suficientemente constante $y$ distinta de otras formas para poder ser definida; $y$, si es definible, si las diferencias son lo suficientemente 
importantes para merecer un nombre específico ... La única distinción entre especies y variedades bien marcadas es que se sabe, o se cree, que las últimas están actualmente conectadas por gradaciones intermedias ... En resumen, tendremos que considerar las especies del mismo modo que esos naturalistas tratan los géneros, admitiendo que los géneros no son más que combinaciones artificiales hechas por conveniencia. Puede que esto no sea una perspectiva agradable, pero al menos nos habremos liberado de la vana búsqueda de la inencontrada e inencontrable esencia del término especie (Darwin citado por Mayr, 1992: 43).

Sin embargo, sea cual fuere el concepto de especie que tenía Darwin, uno de sus grandes logros fue el hecho de analizar el cambio en grandes poblaciones, en un amplio lapso de tiempo y en una extensión geográfica considerable. Aunque no usó métodos de análisis estadísticos, sí se puede inferir un tratamiento de este tipo en sus conclusiones. Lamarck, por el contrario, no pudo ver más allá de individuos esforzándose por transformarse. Aquí es donde puede verse un gran punto de discrepancia entre las dos teorías, ya que las ideas de Lamarck son consideradas como transformistas (Jacob, 1986). El transformismo de Lamarck se caracteriza por el hecho de que los seres van de lo simple a lo complejo en una escala preestablecida y en donde está implícito el esfuerzo de los organismos. Mientras que en el caso de Darwin, se puede hablar de un evolucionismo (aunque él casi no haya usado la palabra evolución), puesto que grandes poblaciones de individuos (¿especies?) se transforman en el espacio y en el tiempo para dar origen a otro tipo de población, sin la intervención de fuerzas sobrenaturales.

De lo expuesto en este aparte podemos concluir que si bien la concepción de especie en Darwin es muy difícil de definir, él concibe poblaciones de individuos que, por efecto de las variaciones individuales, por la selección natural y en un lapso enorme de tiempo evolucionan para transformarse en otras, en donde predominan las variedades seleccionadas, es decir, que sí hay un límite (reproductivo) entre dichos grupos de organismos. Mientras que para Lamarck no hay poblaciones, no hay especies. Así mismo, es importante ver que las dificultades de los científicos para ponerse de acuerdo en las definiciones nos muestran lo arbitrario del pensamiento científico, y que si hay un mundo externo y objetivo igual para todas las personas, ¿por qué es tan difícil hablar de lo mismo en los mismos términos?

\section{LAS EXTINCIONES}

Lamarck tiene muchos problemas para concebir la extinción de las razas (especies), puesto que los fósiles representan una prueba de la transformación de los organismos: si se hallan fósiles de animales que hoy no podemos ver es porque éstos se han transformado, pero no es una prueba de que hayan desaparecido. 
Lamarck llama como especies perdidas a lo que nosotros llamamos especies extinguidas. Sin embargo, Lamarck dice que si hay especies perdidas, éstas están representadas por grandes mamíferos que el hombre ha cazado indiscriminadamente hasta hacerlos desaparecer; de ahí que las especies marinas sean las más invulnerables a la extinción, pues allí están a salvo de los humanos. Si Lamarck hubiera aceptado la extinción de algunas especies estaría negando el equilibrio de la naturaleza que tanto defendía. Esta postura lamarckiana es una evidente oposición a los cataclismos de Cuvier.

Para Darwin, por el contrario, la extinción de las especies se convierte en una prueba a su favor, ya que es una evidencia de que algunas especies no han superado la prueba de la selección natural, no han dejado descendencia y han desaparecido para siempre. De otro lado, este naturalista usa la imperfección del registro geológico (fósiles) para argumentar la ausencia de formas intermedias: los eslabones perdidos que tanto le pedian sus detractores. Mientras que Darwin usaba la imperfección de los registros fósiles para argumentar la inexistencia de formas intermedias, Lamarck usaba la imperfección de las colecciones de los museos para defender la gradación entre los organismos, para conformar su escalera hacia la perfección.

\section{EL ORIGEN DE LA VIDA}

Lamarck postula el origen de todos los organismos en la doctrina de la genera- ción espontánea, que consiste en que los seres imperfectos, por ejemplo los infusorios, se generan a partir de la materia inerte, como el fango de un charco. Una vez que se producen, estos individuos empiezan a ascender hacia la perfección, pero paulatinamente. Según él, ello ocurre en la actualidad (de él), es decir, que los seres más imperfectos pueden ser los más recientes, porque este proceso no culmina. Como sabemos, la idea de la generación espontánea fue desbaratada por Pasteur, pero eso es otra historia.

En cuanto a Darwin, no hay en sus obras una idea acerca de la concepción que él tenía sobre el tema, pero en una carta escrita a un colega en 1871 se puede percibir una aproximación a la idea que tenemos en la actualidad:

Se ha dicho a menudo que la totalidad de las condiciones necesarias para la formación del primer organismo vivo son las que presenciamos hoy día y no pueden haber sido otras que las actuales. Sin embargo, si pudiéramos concebir (iy cuán gran suposición implica ese "si"!) la posibilidad de la formación química de un compuesto protéico, en algún estanque cálido y pequeño, que contuviera toda clase de sales amónicas y fosfóricas, que recibiera luz, calor, electricidad, etc., compuesto que una vez formado podría sufrir otros cambios posteriores de mayor complejidad, en las condiciones actuales ese material sería devorado o absorbido al instante, lo cual no habría sido el caso previamente a la formación de 
los primeros seres vivos (Darwin, citado por Alemañ, 1996: 45-46).

Aunque los dos conciben el origen de la vida en la materia inerte, Darwin asume este hecho como ocurrido una sola vez y hace mucho tiempo (esas condiciones han cambiado) mientras que Lamarck dice que el origen de seres imperfectos sigue ocurriendo indefinidamente y por doquier.

\section{LA FORMA DE PROCEDER}

Lamarck fue un naturalista de museo y, aunque se ufanaba de ser un gran observador, parece ser que no lo era tanto (prueba de ello es su ejemplo de las patas delanteras de la jirafa). Además, sus observaciones eran referidas a las muestras o colecciones a su disposición en el Museo de Historia Natural. De otro lado, el método que argumentaba seguir era el deductivo:

El verdadero medio, en efecto, de llegar a conocer bien un objeto, hasta en sus más mínimos detalles, consiste en considerarlo en su totalidad (...) Después se divide el objeto de que se trata en partes principales, para estudiarlas y considerarlas separadamente (...) Terminadas tales indagaciones, se procura deducir las consecuencias de ellas (...) Únicamente por este método de análisis progresan todas las ciencias (Lamarck, 1986 [1809]: XX-XXI).

Por su parte, Darwin era un naturalista de campo, que exploraba la naturaleza in situ, que tomaba muestras, las analizaba, hacía mediciones. En cuanto al método que decía utilizar era el inductivo. Darwin dice en su autobiografía que trabajó bajo los principios baconianos y que sus observaciones no estaban hechas a partir de una teoría. Sin embargo, en el primer párrafo de la introducción de El origen dice que al llegar a Inglaterra se le ocurrió reunir datos y reflexionar sobre ellos para trabajar sobre la cuestión del origen de las especies (Ruiz y Ayala, 1998: 33). Desde el punto de vista de Ruiz y Ayala (1998: 34), esta incongruencia entre el hacer y el decir de Darwin se debe, de un lado, a que ese método (el baconiano) era el usado por la ciencia del momento (si una teoría no se había producido bajo este método, no era científica) y, de otro lado, porque en su época el término hipótesis era equivalente a simple especulación.

Sin duda, Darwin se planteó unas hipótesis que luego, con la ayuda de la experimentación y el análisis, las fue encajando en una teoría. No partió de la simple observación: ya tenía unas ideas (hipótesis) sobre las cuales trabajar.

\section{Conclusiones}

Aunque podría pensarse que entre Lamarck y Darwin hay una continuidad, debido a que sus teorías comparten algunos conceptos, lo que se ha defendido y demostrado a lo largo de este ensayo es que en los aspectos fundamentales de las dos teorías (principalmente en los mecanismos propuestos) y las formas de proceder de estos naturalistas hay una verdadera ruptura, una discontinuidad. 
Dicho de otro modo, Darwin no formuló su teoría sobre las bases lamarckianas, sino que las contradijo para establecer sus conclusiones.

Pese a que Darwin creyera en la herencia de los caracteres adquiridos, este hecho no constituyó una parte central de su teoría, lo que sí ocurrió en el mecanismo lamarckiano. Pero hay que tener en cuenta que dicha doctrina hacía parte del mundo científico de la época y el trabajo de Mendel aún no había sido reconocido por la comunidad científica. En cuanto al tiempo, tampoco hay diferencias fundamentales, ya que estos dos naturalistas argüían a espacios de tiempo casi infinitos, para que sus mecanismos pudieran llevarse a cabo.

En síntesis, ¿cuál es la verdadera diferencia entra las teorías de Lamarck y Darwin?

Retomando a Jacob (1986), la teoría lamarckiana es una doctrina transformista, lo cual quiere decir que hay una dirección, un plan, para que la transformación se lleve a cabo, que dicha transformación va de lo simple a lo complejo y que hay una fuerza interna (¿vitalismo?) que empuja a los individuos hacia la complejidad, hacia la perfección. Cuando un nuevo ser se forma, ya hay un espacio en la gran cadena para él y se instala allí, es decir, que la intención precede a la realización. Todo ser que se forma, encaja perfectamente en el equilibrio de la naturaleza. La transformación en Lamarck es lineal. Si se pudiera resumir en una palabra, la teoría de Lamarck está representada por la necesidad.
Por su parte, la teoría darwiniana está enmarcada bajo el concepto de la contingencia, es una teoría del azar, pero no de un azar ciego, sino mediado por la selección. De acuerdo con Darwin, primero aparecen los organismos y es la naturaleza la que selecciona cuáles variedades sobreviven y dejan descendencia: "la realización es anterior a todo juicio de valor sobre la calidad de lo que está realizado" (Jacob, 1986: 175). Además, la evolución en Darwin es más horizontal: se hace énfasis en la distribución geográfica.

Para finalizar, quisiera citar un aparte de Lamarck en su Filosofía zoológica (1986 [1809]: 260), a manera de reivindicación de este naturalista:

Habré conseguido el objetivo que me propongo si los amantes de las ciencias naturales encuentran en esta obra algunos puntos de vista y algunos principios útiles, si las observaciones que he expuesto en ella se confirman o son aprobadas por los que han tenido ocasión de ocuparse de estos mismos temas y si las ideas que harán que nazcan, sean las que sean, pueden hacer avanzar nuestros conocimientos o ponernos en camino de llegar a las verdades desconocidas. 


\section{Bibliografía}

Alemañ, R. (1996). Evolución y creación. Barcelona: Editorial Ariel.

Castro, J.A. (2004). Los orígenes del Origen de las especies. En Tecné, Episteme y Didaxis, No. 16, pp.47-63. Bogotá: Universidad Pedagógica Nacional.

Coleman, W. (1985). La biología en el siglo XIX. México: Fondo de Cultura Económica.

Darwin, C. (1986) [1859]. El origen de las especies. Bogotá: Editorial Época.

Dawkins, R. (1988). El relojero ciego. Barcelona: Labor.

Giordán, A., y otros. (1988). Conceptos de biología. Tomo 2. Barcelona: Labor.

Gould, S. J. (1986). El pulgar del panda. Barcelona: Ediciones Orbis.

Gould, S. J. (1993a). La sonrisa del flamenco. Barcelona: Crítica.

Gould, S. J. (1993b). «Brontosaurus» y la nalga del ministro. Reflexiones sobre historia natural. Barcelona: Crítica.
Gould, S. J. (1994). Ocho cerditos. Barcelona: Crítica.

Gould, S. J. (1999). La montaña de almejas de Leonardo. Ensayos de historia natural. Barcelona: Crítica.

Jacob, F. (1986). La lógica de lo viviente. Barcelona: Salvat.

Lamarck, J. B. (1986) [1809]. Filosofía zoológica. Barcelona: Editorial Alta Fulla.

Mayr, E. (1992). Una larga controversia: Darwin y el darwinismo. Barcelona: Crítica.

Ruiz, R., y Ayala, F. (1998). El método en las ciencias. Epistemología y darwinismo. México: Fondo de Cultura Económica.

Smith, C. U. M. (1997). El problema de la vida. Madrid: Alianza Editores.

Vorzimmer, P. (1968). Darwin and Mendel: the historical connection. Isis, Vol. 59, No. 1, pp. 77-82.

ARTíCULO RECIBIDO: 28-07-2004

Y APROBADO: 17-06-2005 